\title{
1 The use of finger foods in care settings: An integrative review.
}

2 Milly Heelan ${ }^{1}$, Jacqui Prieto ${ }^{1}$, Helen Roberts ${ }^{2}$, Naomi Gallant ${ }^{1}$, Colin Barnes ${ }^{3} \&$ Sue Green ${ }^{4}$

$3{ }^{1}$ School of Health Sciences, University of Southampton, University Road, Southampton, $4 \mathrm{SO} 17$ 1BJ

$5 \quad{ }^{2}$ Faculty of Medicine, University of Southampton, Southampton General Hospital Mailpoint

6 807, Southampton So16 6YD

$7 \quad{ }^{3}$ Research and Improvement Team, St Marys Community Health Campus, Office 13 PEC,

8 Milton Road, Portsmouth, PO3 6AD

$9{ }^{4}$ Faculty of Health and Social Sciences, Bournemouth University, Christchurch Road,

10 Bournemouth, BH1 3LH

11 Key words: Finger foods; care setting; adults; integrative review

12 Corresponding author: Milly Heelan, School of Health Sciences, University of

13 Southampton, University Road, Southampton, SO17 1BJ Tel: 07708943678 Email:

14 a.r.heelan@soton.ac.uk

15 Authorship: MH, SG, HR, CB were involved in the design of the review, developing the 16 protocol extracting and appraising study methodologies. $\mathrm{MH}$ and NG had a lead role in 17 identifying articles for inclusion. $\mathrm{MH}, \mathrm{SG}, \mathrm{JP}, \mathrm{HR}$, and $\mathrm{CB}$ contributed to data analysis and with the drafting of the paper. All authors named in the paper agreed the final version of the manuscript.

Conflict of interest : The authors declare no conflict of interest.

Funding statement : This review was completed as part of a clinical doctoral research fellowship for the first author (MH). The research fellowship is funded through an educational grant by Medirest, a division of Compass Group UK and Ireland, however this company had no input with the study design or analysis.

Acknowledgement: The authors would like to acknoweldge Vicky Fenerty, Research Engagement Librarian, for her support in developing the search strategy. 


\section{Abstract}

28 Background: Reduced food intake is prevalent in people in residential and hospital care settings. Little is known about the use of finger-foods, (foods eaten without cutlery), to increase feeding independence and food intake. The Social Care Institute for Excellence ${ }^{(1)}$ recommends the use of finger foods to enable mealtime independence and to prevent loss of dignity and embarrassment when eating in front of others. The aim of this review is to identify and evaluate existing literature regarding the use and effectiveness of finger foods among adults in health and social care settings.

Methods: An integrative review methodology was used. A systematic search of electronic databases for published empirical research was undertaken in October 2018. Following screening of titles and abstracts, the full text of publications, which investigated outcomes associated with the provision of finger foods in adult care settings, were retrieved and assessed for inclusion. Two independent investigators conducted data extraction and quality assessment using Critical Appraisal Skills Programme checklists. Thematic analysis was used

41 to summarise the findings.

42 Results: Six studies met the inclusion criteria. Four themes were identified: Finger food 43 menu implementation; Importance of a team approach; Effect on nutrition and Influence on 44 wellbeing. Study designs were poorly reported, with small sample sizes.

45 Conclusions: There is some evidence that provision of finger foods may positively affect 46 patient outcomes in long-term care settings. There is a paucity of research evaluating the use 47 of a finger food menu in acute care settings, including economic evaluation. Future high 48 quality trials are required. 
The aging population living with multiple co-morbitities, for example dysphagia, stroke and dementia is increasing ${ }^{(2)}$. Older people, particularly those living in residential care settings and those admitted to hospital, are at risk of reduced oral food intake and malnutrition ${ }^{(3)}$. Being under- nourished can cause loss of muscle mass and weakness, together with other physiological effects, including increased susceptibility to infection and delayed wound healing ${ }^{(2)}$. It can impact on mental well-being and lead to reduced quality of life as a result of increased dependence on others ${ }^{(4 ; 5)}$. Malnutrition is associated with increased costs to national health services as a result of extended and more frequent hospital stays and multiple General Practicitioner (GP) visits ${ }^{(6)}$.

Reduced food intake in institutional care can be due to a number of complex factors, including the environment and the patient ${ }^{(7)}$. Environmentally, staff shortages reducing access to mealtime assistance, limited choice, unappealing food and mealtime interruptions can lead to a patient refusing food. Patient factors relating to eating difficulties can be associated with older age ${ }^{(8)}$ as well as specific diseases such as dementia or stroke. People with dementia experience change in cognition, which can cause difficulties recognising food or cutlery, uncoordinated transfer of food from the plate to the mouth and distraction during the mealtime task ${ }^{(9)}$. People after stroke experience physical changes such as hemiparesis, limb apraxia or visual disturbances, which can cause difficulty manipulating cutlery or transferring food from the plate to the mouth ${ }^{(10 ; 11 ; 12)}$, alongside embarrassment when eating in view of others ${ }^{(13)}$.

The need to improve food intake in care settings has been acknowledged internationally, resulting in the publication of guidelines ${ }^{(14)}$. Guidelines include various proposed strategies to improve intake in older adults and particularly adults with dementia, however little is known about the effectiveness of these strategies to improve oral intake ${ }^{(15 ; 16 ; 17)}$. Evidence based recommendations for healthcare promote the provision of adequate support for people who are unable to eat independently ${ }^{(18)}$ and offering food that is appropriate for the person, using a food first approach ${ }^{(14)}$. Despite this, relatives of older people frequently report inadequate amount of appropriate food and lack of support for people unable to feed themselves ${ }^{(19 ; 20)}$. The European Society for Clinical Nutrition and Metabolism (ESPEN) clinical recommendations ${ }^{(21)}$ suggests using finger foods for older adults due to their limited cost and low risk, although the supporting evidence for this intervention is sparse. 
For the purpose of this integrative review, finger foods are defined as foods presented in a form that are easily picked up with the hands and transferred to the mouth without the need for cutlery. Finger foods are considered easier to eat as they do not require manipulation with cutlery ${ }^{(22)}$. Typically, a finger food menu includes small sandwiches, pieces of quiche, cut up vegetables and cake slices or foods presented in bite sized portions, for people managing regular textured foods ${ }^{(23)}$.

The Social Care Institute for Excellence ${ }^{(1)}$ recommends the use of finger foods to enable mealtime independence and to prevent loss of dignity and embarrassment when eating in front of others ${ }^{(24)}$. For people after stroke or with cognitive impairment, finger foods have the potential to support participation and to increase independence at mealtimes ${ }^{(21 ; 25 ; 26)}$. Potential benefits of using finger foods are enhancement of nutritional intake and maintenance of weight ${ }^{(21 ; 27)}$. Additionally, finger foods are described as a more flexible approach to dining ${ }^{(28)}$. They can be used as a portable alternative to a plated meal and can be eaten "on the go" (22).

No previous high quality reviews have purposefully addressed the use of a finger food menu with older adults in care settings. NHS hospital trusts have implemented finger foods as part of a multimodal approach to nutritional intervention, without evidence showing that they singularly have a positive impact on patients ${ }^{(29)}$. Locating and reviewing the literature to identify which finger foods are most appropriate, which groups would benefit and the cost effectiveness of the intervention would inform future research and support clinical practice, guiding decisions regarding resource allocation. Therefore, the aim of this review was to locate and synthesise empirical published literature on the use of finger foods in adults in care settings.

\section{Materials and methods}

An integrative review methodology allows full understanding of a phenomenon ${ }^{(30)}$. It supports the objective critique and summary of selected quantitative and qualitative research studies, as opposed to a systematic review which addresses a distinctive clinical question and evaluated the effectiveness of an intervention ${ }^{(31)}$. This integrative review follows the five steps outlined by Souza et al. ${ }^{(32)}$ : definition of the guiding question, a detailed and systematic search of the literature, data extraction, critical analysis of included publications and interpretation and synthesis of results.

\section{Selection criteria}


Eligible studies were selected through predefined inclusion criteria developed using the

114 PICOST tool (Population, intervention, comparator, outcome, setting, type) ${ }^{(33)}$. Studies were included if, (i) the sample population included adults aged 18 years or above, (ii) the study involved use of finger foods, including an increase in finger foods offered, (iii) Any comparator was present, or none at all, (iv) Any subsequent outcomes were used, (v) the study was conducted in any institutional setting (e.g. long-term care centres, assisted living residence, residential homes, nursing homes, acute hospital ward) (vi) was an example of empirical research. Review publications were not included, as the aim was to find empirical evidence.

\section{Search strategy}

123 Databases were searched using a wide range of pre-defined search terms developed with the assistance of a medical librarian and combined using Boolean operators (And/Or/Near) and MeSH (Medical Subject Heading) terms. This aimed to retrieve the widest scope of publications possible across different platforms. In addition, reference lists of selected publications were searched. In attempt to review the most robust publications, grey literature was not included in this search.

Databases searched to October 2018 included MEDLINE, EMBASE, CINAHL Plus ${ }^{\circledR}$ with Full Text (1937-2018), Psych INFO (1880-2018), Web of Science, Cochrane and Ahmed. No language restrictions were placed during the search. Search terms included: adult, patient, elderly, senior, geriatric, dementia, Alzheimer's, neurocognitive impairment, neurocognitive decline, finger food, buffet, utensil less, menu modification, mealtime intervention, dementia diet and eating with hands or fingers.

The inclusion criteria were used by two investigators ( $\mathrm{MH}$ and $\mathrm{NG}$ ) to screen title and then abstracts initially. Full texts of publications that appeared to be relevant were retrieved for further consideration by three investigators ( $\mathrm{MH}, \mathrm{NG}, \mathrm{SG})$.

\section{Data extraction and quality}

Selected publications were read multiple times to ensure familiarity. Data were extracted using a pre-prepared and piloted instrument based on the data extraction table by Souza et al. (CASP) tool for the study design ${ }^{(34)}$. This tool supports systematic evaluation of published papers, considering validity, credibility, relevance and results of papers ${ }^{(34)}$. Results of the 
CASP tool were discussed and agreed with multiple authors (SG and MH). None of the publications included met all the criteria assessed by the CASP appraisal form. However, it was not possible to assess whether the publications omitted these key components or whether it was simply not reported by the authors, despite attempts to contact authors.

\section{Data synthesis}

Primary data sources were coded, categorized and synthesised using a systematic approach in accordance with guidelines for preparing an integrative review ${ }^{(30)}$. Due to the small number of publications found, it was not necessary to subgroup papers. Initial codes were derived inductively from publications, using descriptive codes to simplfy and sort data into manageable data forms. Next, these descriptive codes were displayed in a visual matrix to observe patterns and themes. Codes were analysed iteratively by clustering descriptive codes into overarching themes and comparing and contrasting codes. These overarching themes were discussed and agreed with the other authors. All relevant studies identified were included in the thematic analysis regardless of quality.

\section{Results}

\section{Descriptive findings}

Six publications were included in the final selection. Figure 1 summarises the selection process using the preferred reporting items for systematic review and meta-analysis (PRISMA) flow diagram, including reasons for exclusion.

Table 1 provides a summary of the publication characteristics. Publications reported studies undertaken in long-term care settings in the United States of America ${ }^{(35 ; 36)}$, the United Kingdom ${ }^{(24 ; 27 ; 37)}$ and France ${ }^{(38)}$. None described the use of a finger foods in acute care settings. Study designs varied including, observational studies ${ }^{(36 ; 37 ; 38)}$, a pilot study ${ }^{(35)}$, a case-study ${ }^{(24)}$ and a reterospective study ${ }^{(27)}$, but did not include randomised controlled trials. Sample sizes were generally small ranging from six participants ${ }^{(24)}$ to 114 participants ${ }^{(38)}$ using a range of outcome measures.

All participants included had a diagnosis of dementia or other psychiatric conditions. Participants presented with a range of physical and cognitive eating difficulties, which were 172 attributed to their cognitive impairment. These included difficulties using utensils $(24 ; 27 ; 35 ; 36$; 37), for example poor hand or finger control, tremor and limited concentration or high level of distractibility ${ }^{(36)}$. 
176 Assessment using the CASP case control critical appraisal tool indicated that two

177 publications reporting quantitative findings were of low quality ${ }^{(27 ; 35)}$. Soltesz and Dayton ${ }^{(27)}$

178 used a control group, which differed in key characteristics to the intervention group. The

179 control group comprised of 11 residents consuming a modified pureed diet, and an intervention group of 43 residents with no swallowing difficulties eating a normal diet. In addition, confidence intervals were not provided for key outcomes, giving no indication of variability ${ }^{(27)}$.

183

In the study by Jean ${ }^{(35)}$, participants acted as their own control groups, in a pre-post study design. No confounding factors were reported, making it difficult to attribute maintenance or increase in weight to the finger food menu intervention ${ }^{(35)}$. Additionally, Jean ${ }^{(35)}$ presented results using only descriptive statistics, which makes it difficult to generalise the results found and places at risk of external validity. Based on the CASP case control checklist, Pouyet et al. ${ }^{(38)}$ study satisfied most criteria of the three studies, however being the only study of its kind, reporting on attractiveness of pureed finger foods, limits the external validity.

The studies employing a qualitative methodology were assessed as low quality ${ }^{(24 ; 36 ; 37)}$. Ford (37) did not report sufficient detail of the study methodology or findings. Barratt et al. (24); Nangeroni and Pierce ${ }^{(36)}$ did not adequately consider the researcher and participant relationship, ethical considerations and included unclear statements of findings and credibility. Limited information regarding the recruitment strategy or reasons for population recruited, makes it difficult to establish target sample for all studies

\section{Meta synthesis}

Four main themes were identified inductively through thematic analysis: (i) Finger food menu implementation; (ii) Importance of a team approach; (iii) Effect on nutrition (vi) Influence on wellbeing.

\section{Finger food menu implementation}

Included publications defined finger foods as food that did not require cutlery ${ }^{(27 ; 37 ; 38)}$, or could be eaten easily with the hands ${ }^{(24 ; 36 ; 38)}$. Generally, finger foods offered were considered appropriate for residents eating normal or regular textured foods ${ }^{(23)}$ and with no evidence of oropharyngeal dysphagia. However, Barratt et al. ${ }^{(24) ;}$ Nangeroni and Pierce ${ }^{(36) ;}$ Pouyet et al. 
${ }^{(38)}$ used softer foods and pureed forms of finger food ${ }^{(38)}$ to support older people with dysphagia or difficulties chewing. Pouyet et al. ${ }^{(38)}$ showed the pureed finger foods were generally well accepted by adults with Alzheimer's disease, with reports that shape was not an influence on food attractiveness ${ }^{(38)}$. The authors, however, did consider shape as important to support manipulation with the hands.

Details of the specific implementation of a finger foods varied. In two studies, finger foods were offered alongside the normal menu to increase variety of food offered ${ }^{(27 ; 36)}$. Soltesz and Dayton ${ }^{(27)}$ added extra finger foods to the existing menu, however the overall number of finger foods increased minimally, leading to difficulties comparing the control and intervention group. This contrasts with other publications, where a finger food menu was developed to replace the standard menu offered over lunch and dinner times ${ }^{(24 ; 35)}$, or offered as smaller, more frequent meals ${ }^{(37)}$. None of the publications reported difficulties with intervention fidelity and suggested no additional staff or additional food items were required (27). Success with using finger foods was supported using simple and easy foods for staff to make ${ }^{(35)}$.

The cost of implementing a finger food menu was considered by Barratt et al. ${ }^{(24) ;}$ Soltesz and Dayton ${ }^{(27) ;}$ Jean ${ }^{(35)}$. However, none reported a robust economic evaluation, resulting in conflicting results. Soltesz and Dayton ${ }^{(27)}$ suggested the implementation of a finger food menu cost no more than the provision of standard foods and Jean ${ }^{(35)}$ suggested that high energy and protein supplements were discontinued in $25 \%$ of participants receiving a finger food menu giving a cost saving. Conversley, in a later study Barratt et al. ${ }^{(24)}$ described an increase on cost per person to implement the finger food menu.

\section{Importance of a team approach}

Collaboration between clinical and catering teams to support the provision of a finger food menu was a common theme arising in three papers ${ }^{(24 ; 27 ; 35)}$. Despite catering services often perceived as non-clinical services, their involvement in ensuring food was presented in a way that patients could access allowed observable changes in clinical outcomes ${ }^{(24)}$. In publications showing increased costs for providing finger foods, agreements between budget holders - often clinical managers, commissioning services and catering teams - is required to justify the need for this intervention ${ }^{(24)}$. Staff training in understanding the need and rationale of finger foods was one approach influencing maintenance and success of implementing the intervention across departments ${ }^{(35 ; 37)}$. 
238 Barratt et al. ${ }^{(24) ;}$ Soltesz and Dayton ${ }^{(27)}$ described collating feedback from the clinical and catering team to support the development and implementation of the finger food, however little detail was given about the changes made and how this data was collected.

241

242

\section{Effect on nutrition}

Nutritional outcomes were measured in only three studies by assessing food intake via food chart reviews, plate waste observations and changes in weight ${ }^{(35 ; 37)}$. Increased nutritional intake and weight maintenance during the finger food menu intervention period was demonstrated in all three studies ${ }^{(27 ; 35 ; 37)}$. Full description of the menu offered with nutritional values was not provided, therefore, although there was an increase in weight of food consumed, the nutritional value of the foods eaten could not be evaluated. Ford ${ }^{(37)}$ suggested that changes in nutritional status could affect medical status, however an explanation as to how medical status will change was not included.

\section{Influence on wellbeing}

The fourth theme describes the improvement in wellbeing during the implementation of finger foods which was reported in all publications. Wellbeing was measured formally by Barratt et al. ${ }^{(24)}$, using Dementia Care Mapping. Barratt et al. ${ }^{(24)}$ demonstrated an increase in mean wellbeing scores of residents offered a finger food menu which was maintained six weeks after the introduction. However, the small sample size used by Barratt et al. ${ }^{(24)}$ and pre-post study design limits control of confounding variables in the complex long-term care setting and makes it difficult to attribute these findings wholly to the food offered.

Increased independence with eating for people chosing to eat finger foods was described in three studies ${ }^{(24 ; 35 ; 36)}$, despite variation in outcome measures used. Barratt et al. ${ }^{(24)}$ observed an increase in the mean percentage of observations recorded as 'independent feeding' over lunchtime meals. This contrasts to Jean ${ }^{(35)}$ who created a scale which demonstrated 3 of 12 residents became fully independent eating their meal when offered finger foods, despite during the baseline measure being fully dependent with feeding. Nangeroni and Pierce ${ }^{(36)}$ did not provide details of how independence was measured. Within these studies, blinding or reflexive views of the researcher were not described, which increases the risk of bias and makes it difficult to distinguish whether this would lead to a reduced requirement for support by staff and visitors ${ }^{(24)}$.

\section{Discussion}


The aim of this integrative review was to locate and synthesise empirical published literature on the use of finger foods for adults in care settings, to inform future research and support clinical practice and policy decisions.

The lack of high quality trials identified suggests the use of a finger foods with adults is yet to be robustly evaluated. There is some evidence to demonstrate improvement in relevant outcomes, such as food intake, but this has been shown in studies that lacked a control making it difficult to ascertain the cause of the effect shown. The variation in interventions provided across these publications provides additional challenges when comparing outcomes. However, this does highlight the need for a pragmatic approach to future research, considering all stakeholders involved. A study by Cluskey and Kim ${ }^{(39)}$ undertaken in the USA suggested that finger foods are judged by healthcare professionals, working in long term care settings, as being beneficial for residents, cheap and easily implemented in institutions. The limited adverse effects and expense to provide these types of foods means that their use continues to remain in clinical guidelines on nutrition and hydration in geriatrics ${ }^{(21)}$.

Despite guidelines suggesting that finger foods could be used to support people with other conditions, such as stroke ${ }^{(25)}$, all studies focussed on people with cognitive impairment. Ford (37) acknowledged the potential of using a finger food menu to support older adults with a wide range of eating difficulties, including mental health or physical difficulties. An increase in food intake in people with cognitive impairment has been shown in other studies with different presentations of food. In a cross over, randomised controlled trial undertaken in a nursing home, Young et al. ${ }^{(40)}$ demonstrated increased energy intake when high carbohydrate foods were offered in place of a usual meal which was not fully described. Although this study did not aim to evaluate the use of finger foods, it was noted many of the high carbohydrate foods could be defined as finger foods, such as bread with jam, hard boiled egg, muffins and slices of cheese. In addition, greater severity of cognitive deficit and atypical motor behaviour was associated with greater intervention success ${ }^{(40)}$. Young et al acknowledged that in this trial, people with nutritionally controlled diabetes were excluded from the trial. This highlights that the suitability for a finger food diet would need to be assessed individually as the nutritional content and presentation may not meet some people's dietary needs.

None of the research studies in this integrative review conducted a well described economic evaluation, to assess the benefits of individual interventions and to evaluate the best use of 
available resources alongside highest patient satisfaction ${ }^{(41)}$. It is important to reflect the true direct and indirect costs of healthcare interventions, particularly when implementing a change in practice $^{(42)}$.

Interestingly, none of the studies in this review included or explored the views of staff, carers or the recipient of the finger foods, despite suggestions that they may have positive benefits on quality of life and wellbeing. A conference abstract, with no associated published paper, was identified which used a survey methodology to explore residents, caregivers and relatives experiences of providing a finger food menu in a nursing home ${ }^{(43)}$. It appears further in depth research investigating the experience of residents, caregivers and relatives could give further information on the acceptability of this menu ${ }^{(43)}$ to support effective and efficient service delivery ${ }^{(44)}$.

The findings of this review are in agreement with broader reviews on nutritional interventions. Abdelhamid et al. ${ }^{(45) ;}$ Malerba et al. ${ }^{(46)}$ suggest positive outcomes for the use of finger foods, but further need for high quality investigation and well powered randomised control trials. The review by Abdelhamid et al. ${ }^{(45)}$ focussed on interventions to support food intake in people with dementia and included two studies which classified the use of finger foods as a direct dietary intervention ${ }^{(27 ; 35)}$. Adressing the use of multiple dietary interventions meant the review did not focus specifically on the use of finger foods and limited the range of publications found. However, two studies ${ }^{(27 ; 35)}$ were also included in this integrative review and interestingly no studies published later than 2016 were found. The descriptive review by Malerba et al. ${ }^{(46)}$, in France, commented on the use of finger foods for people with dementia in community and home settings. Malerba et al. ${ }^{(46)}$ suggests beneficial outcomes relating to the use of finger foods, for example reduced workload of carers, increased independence and individualised care for people with dementia. Despite useful results, the review did not show a systematic approach to searching the literature or quality critique of publications included.

\section{Strengths and limitations to integrative review}

The range of study designs included in this review and the synthesis of quantitative and qualitative data adds a level of complexity to the review and therefore can introduce bias ${ }^{(30)}$. To ensure the quality of this review, rigorous systematic approaches were used throughout. To reduce bias, two reviewers (MH and NG) screened 347 abstracts for inclusion and 
332 discrepancies were dealt with through discussion. The full texts were chosen following

333 discussion with the other authors of this paper.

\section{Conclusions and future recommendations}

335 The findings suggest that the use of finger foods may increase nutritional intake and enhance

336 independence and wellbeing for adults with cognitive impairment in long term care settings.

337 However, the low quality of the studies included do not provide robust evidence for the

338 effectiveness for using these types of foods in care settings. Therefore results should be

339 interpreted with caution.

340 The review highlights key considerations to implementing a finger food menu within care

341 settings, and a particular need to focus on the use of this menu in hospital settings. Further

342 research is required to suggest whether this intervention is cost effective, feasible and

343 acceptable to be used in acute care settings for older adults.

344 Transparency Declaration: The lead author affirms that this manuscript is an honest,

345 accurate, and transparent account of the study being reported. The reporting of this work is

346 compliant with PRISMA3 guidelines. The lead author affirms that no important aspects of the

347 study have been omitted and that any discrepancies from the study as planned have been

348 explained. 
1. The Social Care Institute for Excellence (2009) Nutritional Care and Hydration. Great Britain: Social Care Institute for Excellence. 2. Wilson L (2017) Malnutrition task force: State of the Nation. http://www.malnutritiontaskforce.org.uk/wpcontent/uploads/2017/10/AW-5625-Age-UK-MTF Report.pdf (accessed 27/03/2019

3. Elia M (2015) The cost of malnutrition in England and potential cost savings from nutritional interventions: British Association for Parenteral and Enteral Nutrition

4. Altman KW, Yu G-P, Schaefer SD (2010) Consequence of dysphagia in the hospitalized patient: impact on prognosis and hospital resources. Archives of Otolaryngology-Head \& Neck Surgery 136, 784789.

5. Forsey A (2018) Hidden hunger and malnutrition in the elderly. http://www.frankfield.co.uk/upload/docs/Hidden\%20hunger\%20and \%20malnutrition\%20in\%20the\%20elderly.pdf (accessed 04/06/18 6. Guest JF, Panca M, Baeyens J-P et al. (2011) Health economic impact of managing patients following a community-based diagnosis of malnutrition in the UK. Clinical nutrition 30, 422-429. 7. Mudge AM, Ross LJ, Young AM et al. (2011) Helping understand nutritional gaps in the elderly (HUNGER): a prospective study of patient factors associated with inadequate nutritional intake in older medical inpatients. Clinical Nutrition 30, 320-325. 8. Pu D, Murry T, Wong MC et al. (2017) Indicators of Dysphagia in Aged Care Facilities. Journal of Speech, Language, and Hearing Research 60, 2416-2426. 9. Chang CC, Roberts BL (2008) Feeding difficulty in older adults with dementia. Journal of clinical nursing 17, 2266-2274. 10. Intercollegiate Stroke Working Party (2016) National clinical guideline for stroke

380 https://www.strokeaudit.org/SupportFiles/Documents/Guidelines/20 16-National-Clinical-Guideline-for-Stroke-5t-(1).aspx (accessed $15 / 06 / 18$ 
11. Poels B, Brinkman-Zijlker H, Dijkstra P et al. (2006) Malnutrition, eating difficulties and feeding dependence in a stroke rehabilitation centre. Disability and rehabilitation 28, 637-643.
12. Wu H-S, Lin L-C (2015) Comparing Cognition, Mealtime

Performance, and Nutritional Status in People With Dementia With or Without Ideational Apraxia. Biological research for nursing 17, 199206.

13. Medin J, Larson J, Von Arbin M et al. (2010) Striving for control in eating situations after stroke. Scandinavian Journal of Caring Sciences 24, 772-780.

14. Department of Health, Jeffrey D (2014) The Hospital Food Standards Panel's report on standards for food and drink in NHS hospitals https://assets.publishing.service.gov.uk/government/uploads/system/ uploads/attachment data/file/523049/Hospital Food Panel May 20 16.pdf (accessed 24/05/19

15. Council of Europe (2003) Resolution ResAP (2003) 3 on food and nutritional care in hospitals.

https://wcd.coe.int/ViewDoc.jsp?id=85747 (accessed 10/04/2019

16. Herke M, Fink A, Langer $G$ et al. (2018) Environmental and behavioural modifications for improving food and fluid intake in people with dementia. The Cochrane database of systematic reviews 7, CD011542-CD011542.

17. Liu W, Cheon J, Thomas SA (2014) Interventions on mealtime difficulties in older adults with dementia: a systematic review. Int J Nurs Stud 51, 14-27. 18. NICE (2017) Nutrition support for adults: oral nutrition support, enteral tube feeding and parenteral nutrition.

https://www.nice.org.uk/guidance/cg32 (accessed 18/11/17 19. Age Concern (2006) Hungry to be heard. The scandal of malnourished older people in hospital England: The Social Care Institute for Excellence.

20. Care Quality Commission (CQC) (2015) Adult inpatient surveys. http://nhssurveys.org/surveys/425 (accessed 12/11/18 
417 21. Volkert D, Beck AM, Cederholm T et al. (2019) ESPEN guideline on 418 clinical nutrition and hydration in geriatrics. Clinical Nutrition 38, $10-$ 41947.

420 22. Crawley H, Hocking E (2011) Eating well: supporting older people 421 and older people with dementia. A Practical guide. Hertfordshire, 422 England: The Caroline Walker Trust.

423 23. IDDSI committee (2016) IDDSI Framework evidence statement.

424 http://iddsi.org/Documents/IDDSIFramework-EvidenceStatement.pdf 425 (accessed 08/05/18

426 24. Barratt J, Gatt J, Greatorex' B et al. (2001) Using finger food to 427 promote independence, well being and good nutrition in people with 428 dementia. PSIGE Newsletter 77, 26-31.

429 25. BDA Association of UK Dieticians (2017) The nutritional and 430 hydration digest. Improving outcomes through food and beverage 431 services.

432 https://www.bda.uk.com/publications/professional/NutritionHydratio 433 nDigest.pdf (accessed 24/05/19

434 26. Volkert D, Chourdakis M, Faxen-Irving G et al. (2015) ESPEN 435 guidelines on nutrition in dementia. Clin Nutr 34, 1052-1073.

436 27. Soltesz KS, Dayton JH (1995) The effects of menu modification to 437 increase dietary intake and maintain the weight of Alzheimer 438 residents. American Journal of Alzheimer's Disease and Other 439 Dementias 10, 20-23.

440 28. Burbidge D (2013) Food for thought: Facilitating independence 441 with finger foods.

442 http://journalofdementiacare.com/dementia finger foods menu/ 443 (accessed 18/09/17

444 29. James J, Gilby L, Pettit J (2017) Transforming nutrition and 445 hydration for people with dementia in hospital - The NOSH project at 446 Imperial College Healthcare NHS Trust. Age and Ageing 46, i1-i22. 447 30. Whittemore R, Knafl K (2005) The integrative review: updated 448 methodology. Journal of advanced nursing 52, 546-553.

449 31. Noble H, Smith J (2018) Reviewing the literature: choosing a 450 review design. Evidence-based nursing 21, 39-41. 
32. Souza MTd, Silva MDd, Carvalho Rd (2010) Integrative review: what is it? How to do it? Einstein (São Paulo) 8, 102-106.

33. Aveyard $\mathrm{H}$ (2010) Doing a literature review in health and social care : a practical guide England: McGraw-Hill.

34. CASP (2017) CASP Checklists. http://www.casp-uk.net/casp-toolschecklists (accessed 19/09/17

35. Jean $L$ (1997) "Finger food menu" restores independence in dining. Health care food \& nutrition focus 14, 4-6.

36. Nangeroni J, Pierce P (1985) A Geriatric Nutrition Program Designed to Improve Independence in Feeding. Hospital \& Community Psychiatry 36, 666.

37. Ford G (1996) Putting feeding back into the hands of patients... 'utensil-less' diets for patients with acute psychiatric conditions. Journal of Psychosocial Nursing \& Mental Health Services 34, 35-39. 38. Pouyet V, Giboreau A, Benattar L et al. (2014) Attractiveness and consumption of finger foods in elderly Alzheimer's disease patients. Food Quality and Preference 34, 62-69. 39. Cluskey M, Kim YK (2001) Use and perceived effectiveness of strategies for enhancing food and nutrient intakes among elderly persons in long-term care. Journal of the American Dietetic Association 101, 111-114.

40. Young KW, Greenwood CE, Van Reekum R et al. (2005) A randomized, crossover trial of high-carbohydrate foods in nursing home residents with Alzheimer's disease: associations among intervention response, body mass index, and behavioral and cognitive function. The Journals of Gerontology Series A: Biological Sciences and Medical Sciences 60, 1039-1045.

41. Brent RJ (2004) Cost-benefit analysis and health care evaluations. Cheltenham, UK: Edward Elgar Publishing.

42. McMahon A, Sin C (2013) Introduction to economic assessment. Nursing Management 20, 32-38.

43. Tuinier K, Westrate W, Hoogendoorn J et al. (2014) Finger food. Intervention for patients with eating difficulties. In 43rd Biennial Convention Las Vegas, Nevada, USA STTI. 
485 44. Collins J, Huggins CE, Porter J et al. (2017) Factors influencing 486 hospital foodservice staff's capacity to deliver a nutrition intervention. 487 Nutr Diet 74, 129-137.

488 45. Abdelhamid A, Bunn D, Copley M et al. (2016) Effectiveness of 489 interventions to directly support food and drink intake in people with 490 dementia: systematic review and meta-analysis. BMC Geriatrics 16, 49126.

492 46. Malerba G, Pop A, Rivasseau-Jonveaux T et al. (2015) Feeding a 493 patient with neurocognitive impairment in the hospital and at home? 494 Convenience of finger-food. Nutrition Clinique et Metabolisme 29, 495 197-201. 